\title{
La revue ETP/TPE fête ses dix ans !
}

En 2008, la Société d'Éducation Thérapeutique Européenne (SETE) décidait de créer sa revue scientifique, publiant dans les deux langues de la Société : le français et l'anglais. L'éditeur que nous avons choisi pour porter la revue est EDP Sciences, entreprise elle-même issue de deux sociétés savantes, celles de Physique et de Chimie.

Nous écrivions dans l'éditorial du premier numéro de la revue, en juin 2009:

«...nous avons besoin de promouvoir et d'inventer en permanence cette nouvelle pratique de santé, l'éducation thérapeutique, par une recherche de qualité. Nous avons également besoin de stimuler la réflexion et les riches échanges entre les équipes soignantes. Il y a donc place pour une revue qui s'engage dans ces deux directions ».

Le projet de la revue était de publier des recherches quantitatives et qualitatives, mais aussi de partager des expériences validées, des méthodologies, des innovations, de stimuler la réflexion critique et le débat d'idées sur différents aspects de l'éducation thérapeutique.

Une décennie plus tard, nous pouvons constater qu'ETP/TPE s'est imposée comme une des revues scientifiques internationales reconnue dans ce champ disciplinaire. En témoigne son référencement dans des banques de données internationales comme Scopus, CINHAL, Psychinfo, Crossref et en France : Banques de Données en Santé Publique (BDSP), Haut Conseil de l'Évaluation, de la Recherche et de l'Enseignement (HCERES), Littérature Scientifique en Santé ( LISSA).

En témoigne également le fait que 490 auteurs nous ont fait confiance, au fil de ces années, pour nous soumettre près de 180 articles dont le plus grand nombre est cité dans d'autres publications.

Le problème que nous rencontrons, cependant, est que la majorité des recherches sur l'ETP est encore actuellement proposée à des revues disciplinaires. Ceci s'explique par le faible nombre d'équipes universitaires européennes principalement dédiées à l'ETP, mais aussi, peut-être, en raison du souhait des auteurs, dans chaque discipline, de démontrer les effets de l'ETP. La conséquence en est que pour l'instant, nous n'envisageons pas d'éditer plus de deux numéros par an, la moyenne annuelle des articles publiés par numéro depuis le lancement de la revue se situant à 8 .

Par contre, la politique de la revue est de tenter de raccourcir au maximum le temps d'examen des articles, de sorte qu'en peu de mois les auteurs puissent recevoir analyse et recommandations sur leur article.

L'exigence de qualité scientifique est présente depuis la création de la revue. Celle-ci est «peer reviewed», chaque article étant soumis de façon anonyme à deux réviseurs, également anonymes. Nous veillons à ce que les éventuels conflits d'intérêt soient déclarés, à travers une procédure de questionnaire Pubmed. De surcroît, la revue a adhéré à l'organisation internationale COPE qui promeut l'honnêteté scientifique.

L'analyse des articles publiés dans ETP/TPE montre que les études en représentent $75 \%$, les expériences en ETP, $9 \%$, les mises au point $7,3 \%$ et les analyses, $4 \%$. De ces résultats, nous pouvons conclure que la revue est clairement centrée sur la recherche, alors qu'au départ nous pensions que la description d'expériences en ETP constituerait la moitié des propositions d'articles. Cette recherche est à caractère multiprofessionnel et il faut noter que les articles en langue anglaise constituent près d'un quart des publications, ce qui confirme le caractère international de notre revue.

Les prochains défis qui nous attendent sont le référencement de la revue dans Pubmed, que nous pensons solliciter en 2019 et la question - laissée en suspens pour l'instant - de la mise en «Open Access» de ETP/TPE, si telle est la politique voulue par les autorités européennes pour toutes les revues à caractère scientifique.

Je souhaite terminer cet éditorial par des remerciements sincères : aux auteurs qui nous accordent leur confiance, aux réviseurs, au nombre de 102, qui nous donnent de leur temps pour analyser les articles, aux équipes d'édition de EDP Sciences qui font fonctionner au quotidien la revue avec le souci permanent d'en maintenir la qualité éditoriale, à la SETE et à l'IPCEM qui nous soutiennent par leurs abonnements et enfin, aux fidèles lecteurs sans lesquels cette revue n'aurait pas franchi ce cap des dix ans. 